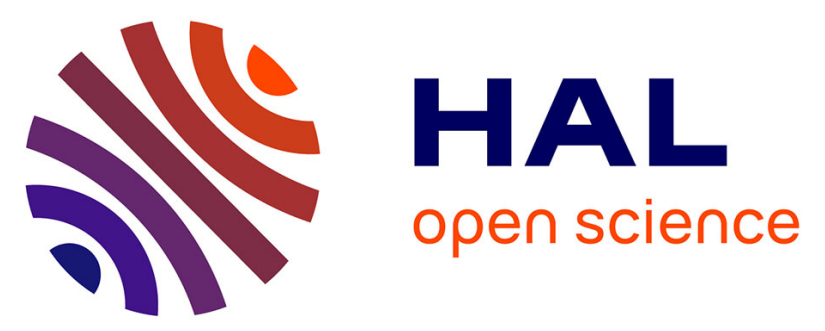

\title{
INVESTIGATION OF THE TRANSMISSION AND STOPPING OF LIGHT IONS PASSING THROUGH A PLASMA TARGET
}

D. Gardes, R. Bimbot, S. Della-Negra, M. Dumail, B. Kubica, A. Richard, M.

Rivet, A. Servajean, C. Fleurier, A. Sanba, et al.

\section{To cite this version:}

D. Gardes, R. Bimbot, S. Della-Negra, M. Dumail, B. Kubica, et al.. INVESTIGATION OF THE TRANSMISSION AND STOPPING OF LIGHT IONS PASSING THROUGH A PLASMA TARGET. Atomic Physics for Ion Driven Fusion 4, 1988, Orsay, France. pp.C7-151-C7-157, 10.1051/jphyscol:1988717. jpa-00228201

\section{HAL Id: jpa-00228201 https://hal.science/jpa-00228201}

Submitted on 1 Jan 1988

HAL is a multi-disciplinary open access archive for the deposit and dissemination of scientific research documents, whether they are published or not. The documents may come from teaching and research institutions in France or abroad, or from public or private research centers.
L'archive ouverte pluridisciplinaire HAL, est destinée au dépôt et à la diffusion de documents scientifiques de niveau recherche, publiés ou non, émanant des établissements d'enseignement et de recherche français ou étrangers, des laboratoires publics ou privés. 


\title{
INVESTIGATION OF THE TRANSMISSION AND STOPPING OF LIGHT IONS PASSING THROUGH A PLASMA TARGET
}

\author{
D. GARDES, R. BIMBOT, S. DELLA-NEGRA, M. DUMAIL, B. KUBICA, \\ A. RICHARD, M.F. RIVET, A. SERVAJEAN, C. FLEURIER*, A. SANBA*, \\ C. DEUTSCH ${ }^{*}$, G. MAYNARD**, D.H.H. HOFFMANN ${ }^{* *}$, K. WEYRICH*** $^{*}$ and \\ H. WAHL** \\ Institut de Physique Nucléaire, F-91406 orsay Cedex, France \\ * GREMI, CNRS, Université de Caen. France \\ * "Laboratoire de Physique des Gaz et des Plasmas, Université \\ Paris-Sud, F-91405 Orsay Cedex, France \\ ** * $\mathrm{MPQ}$-Garching and GSI, F.R.G.
}

\begin{abstract}
Resumé - Les pertes d'Emergies et la transmission cie faisceaux de carbone et de soufre au Tiravers d'un plasma d'hydrogène (2 ev) ont été étudiées expérimentalement. Les mesures de pertes d'énergies dans un gaz d'électrons libres indiquent un pouvoir d'arrêt renforcé conformément aux prédictions théoriques.
\end{abstract}

Abstract - Transmission and energy losses of 2 MeV/u Carbon and Sulphur beams passing through a plasma target, have been extensively investigated. A hydrogen plasma ignited by an electrical discharge was coupled to the orsay Tandem beam accelerator. Fluctuations in beam transmission have been observed and attributed to a magnetic focusing effect generated during the plasma evolution.

Energy loss measurentents were performed on the basis of time of flight techniques and indicate an enhanced stopping power of the plasma relative to its cold matter equivalent.

\section{$\hat{i}-$ INTRODUCTION}

The experimental investigation of the interaction of an heavy ion beam with a dense plasma is a very recent challenge which have been undertaken two years ago at orsay and Darmstadt /ref. 1, 2, $3,4 /$.

The background of these experiments is linked with the inertial confinement fusion (I.C.F). In heavy ion driven inertial fusion, the compression and heating for ignition of the reaction is achieved by means of intense beams striking a spherical pellet containing the D-T fuel.

The figure $/ 1 /$ presents the well known numerical simulation of the Heavy Ion Beam I.C.F. corresponding to the HIBALL project/ref. 5/. This diagram displays some features of the target dynamics and represents a typical I.C.F. scenario. A $5 \mathrm{MJ}, 500 \mathrm{TW} \mathrm{Bi}$ beam converges sphericaly toward the iarget pellet. During the first $10 \mathrm{~ns}$ of the interaction, the outer absorber layer is vaporised and the main part of the interaction process ( nearly $80 \%$ ) takes place in a plasma medium with different temperature and density gradients.

The knowledge of the energy deposition laws in such a medium are thus essential to optimize the beam profile and the target structure. Our experimental goal was to check the validity of theoretical predictions concerning stopping power in hot matter, which indicate an enhanced stopping power relatively to cold matter /ref. $6 /$.

The principle of the measurement is very simple. The plasma target, which has been described by A. FLEURIER, (see contribution to this workshop and ref. 7) is interposed on a beam 1 ine. The Sulphur or Carbon beam delivered by the Orsay Tandem accelerator was bunched with a microstruc.. ture of $2.5 \mathrm{MHz}$, that is to say, one burst every $400 \mathrm{~ns}$. The width of this beam pulse was adjust.ed to $2 \mathrm{~ns}$ and the incident energy $(2 \mathrm{MeV} / \mathrm{u})$, was given by the machine with a relative precision of $10^{-4}$. After interaction, the energy loss was simply deduced from a comparison of the beam pulse energy before and after interaction with the plasma. The velocity of the ions after interaction is measured by a time of flight technique. If we know the density of free electrons inside the plasria were are thus able to deduce the corresponding stopping power. 


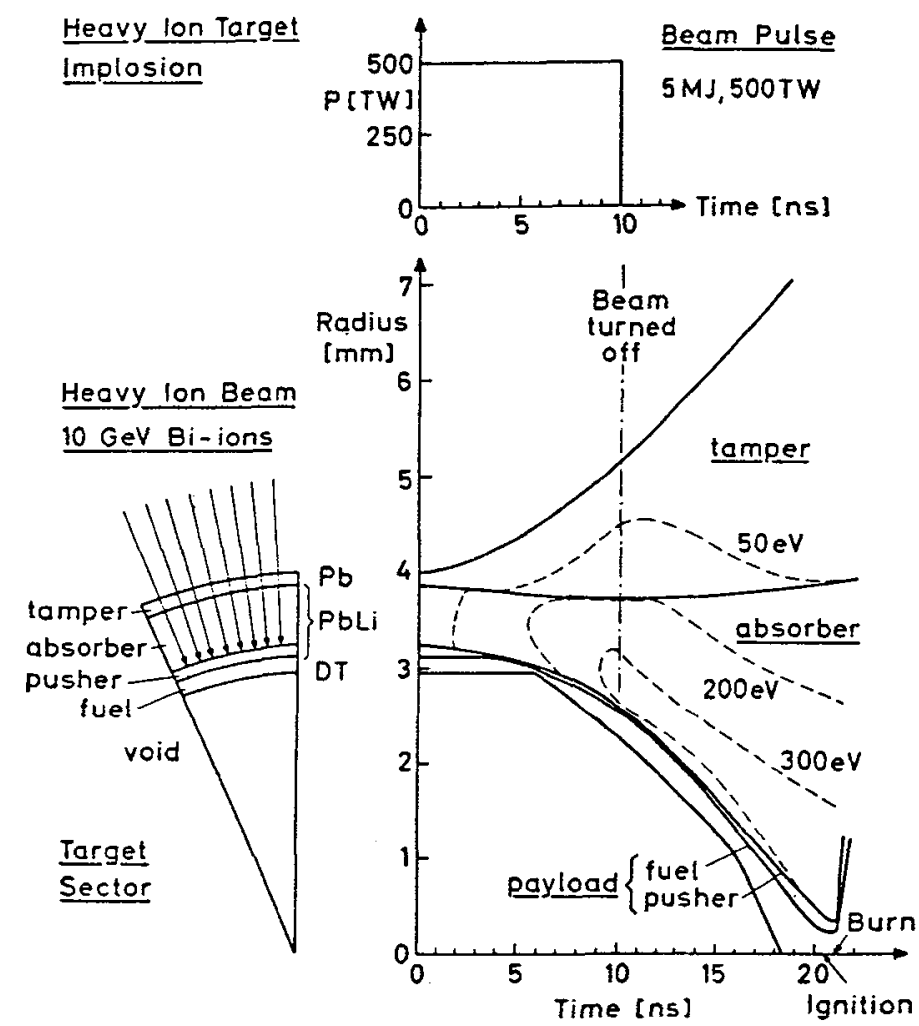

Fig. 1 - Numerical simulation of the implosion of the HIBALL Pellet.

\section{2 - EXPERIMENTAL SET UP}

\subsection{General presentation}

Figure (2) presents the experimental set up. The hydrogen plasma target is connected to the beam line via two long collimators. This is done to suppress any matter on the beam trajectory and to minimize straggling sources in the energy loss process. This imply a powerful differential pumping system. A Faraday cave has been built around the plasma unit including capacitor bank and power supply in order to reduce electromagnetical perturbation during the plasma burst. Figure (2) shows the plasma generator and it's interface with the beam line. Three stages of pumping are successively applied to absorb the hydrogen leakage in order to be able to open the beam 1 ine toward the machine vac um (around $10^{-6}$ Torr).

\subsection{Time of flight measurement}

For the same reasons that we suppressed windows to confine the plasma, it is necessary to avoid any matter on the ion trajectory during the time of fiight measurement. The phase probe offers a very attractive solution to cope with this problem of matterless time pick off. It consists in a copper cylinder connected to a current amplifier. The beam burst passes through the cylinder and it induces by capacity coupling a charge movement which corresponds to upper signal of the figure (3).

The associated current is presented just below, and this bipolar electrical signal is used to perform the time pick off. If the length of the beam pulse fits the length of the copper cylinder we are able to determine the centroid of the beam pulse by detecting the zero crossing of the signal. jpecial attention has been paid to the shielding of the detector against electromagnetical perturoations due to the plasma flash. All the system is carefully grounded and as much as possible we have tried to reduce sources of noise pick up. For instance the low voltage power supply of the 
amplifier is realized electronics elements.

with $\mathrm{Cd}-\mathrm{Ni}$ accumulators. This suppresses long cables coming from external

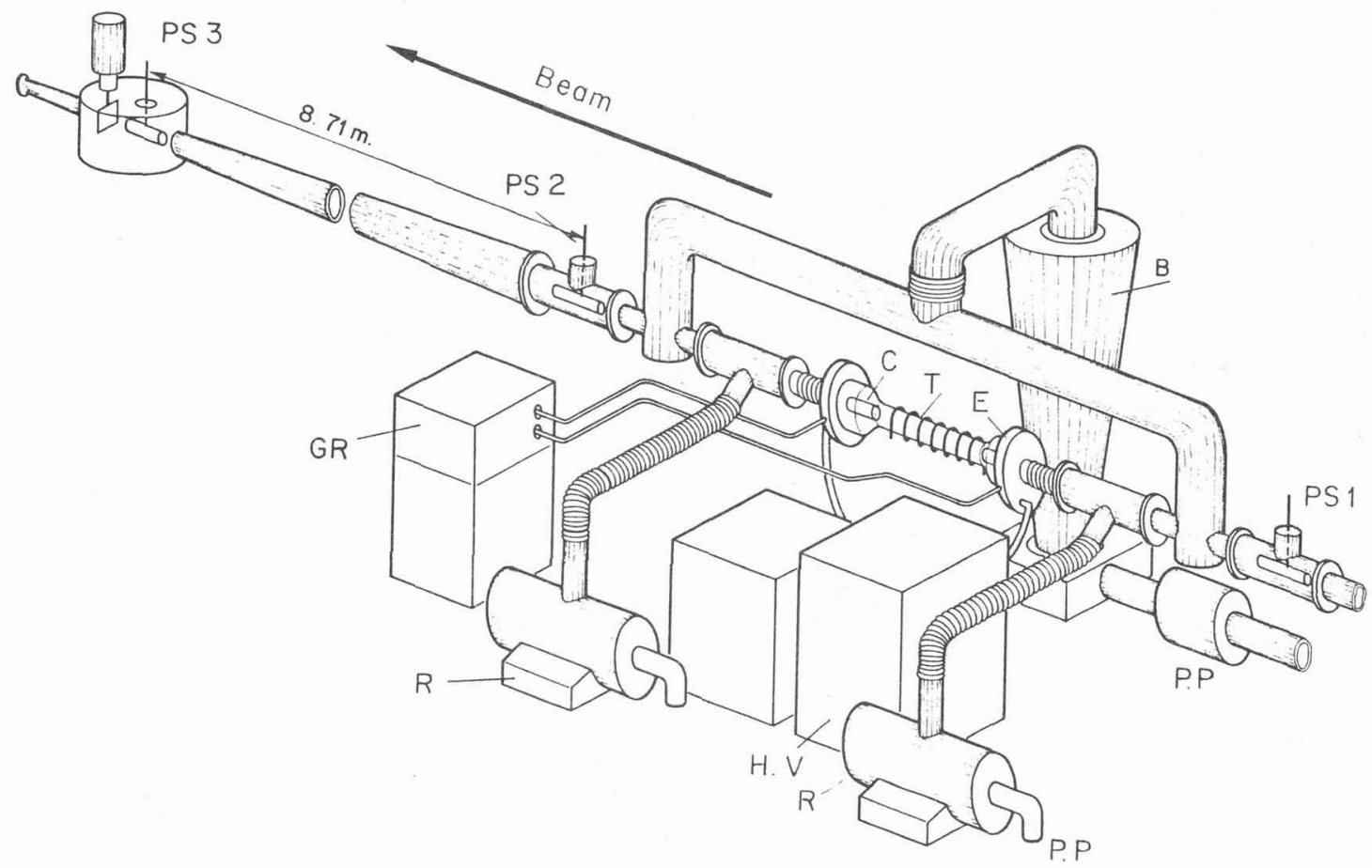

Fig. 2 - Experimental set up. T : plasma target, C : collimators, E : electrodes, G.R. : gas regulation, H.V. : high voltage supply, PP : primary pumps, R : roots, B. : booster : PS1,2,3 : phase probes.

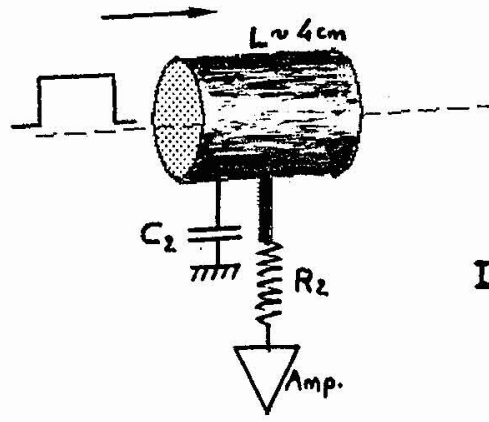

Q

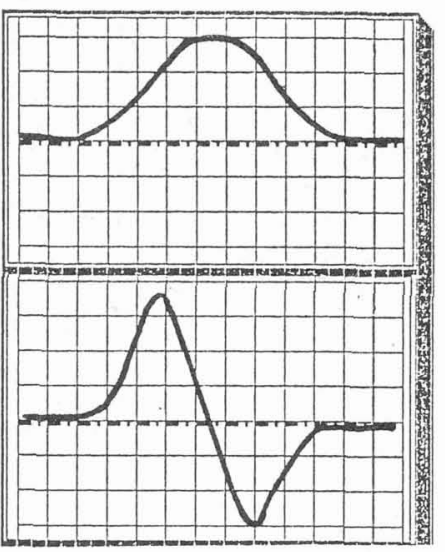

Fig. 3 - Phase probe signal (see text).

$$
2 n
$$


Three phase probes are placed along the beam line. The first one is placed upstream relative to the plasma generator. The detection of the pulse before interaction is used to trigger the acquisition system. A second one is located dowstream just behind the plasma tube and it delivers the start signal for the time of flight analysis. The third phase probe delivers the corresponding stop signal. The distance between these two detectors is $8.7 \mathrm{~m}$.

In this configuration each beam pulse travelling through the experimental set up delivers 3 timing signals. The absolute time shift is obtained by difference between measurements with and without plasma.

Three pics labelled PS1, PS2, PS3 correspond to the signals of the three phase probes. One beam burst is associated with three pics. Between two PS1 or PS2 or PS3 we find the microstructure of the beam : $400 \mathrm{~ns}$. Time difference between PS2 and PS3 corresponds to the velocity of the beam after interaction. The evolution of this time interval between PS2 and PS3 from vacuum measurement to plasma measurement corresponds to the information which is of interest in our case.

A special acquisition device called MultistopConverter (CTN) /ref. 8/ enables us to digitilize up to 255 time records during a chosen period. This time window was fixed to 16 us. During this period about 40 beam bursts pass though the plasma. That is to say that each 400 ns the stopping power is measured and its evolution with time is investigated every $400 \mathrm{~ns}$ during the $16 \mu \mathrm{s}$ of the window time. Then we can proceed to series of plasma shots and if the plasma conditions are reproducible we can expect to obtain the same result for a given period of analysis.

\section{3 - PRELIMINARY}

\subsection{Beam transinission through the plasma}

Two sorts of results have been obtained during these experiments/ref. $4 /$. The first one which was expected on the basis of theoretical predictions (the stopping power measurement). Another one, more surprising, which concerns transmission of the ion beam through the plasma. Figure (4) displays the PS2 detector response. The amplitude of the phase probe signal is proportionnal
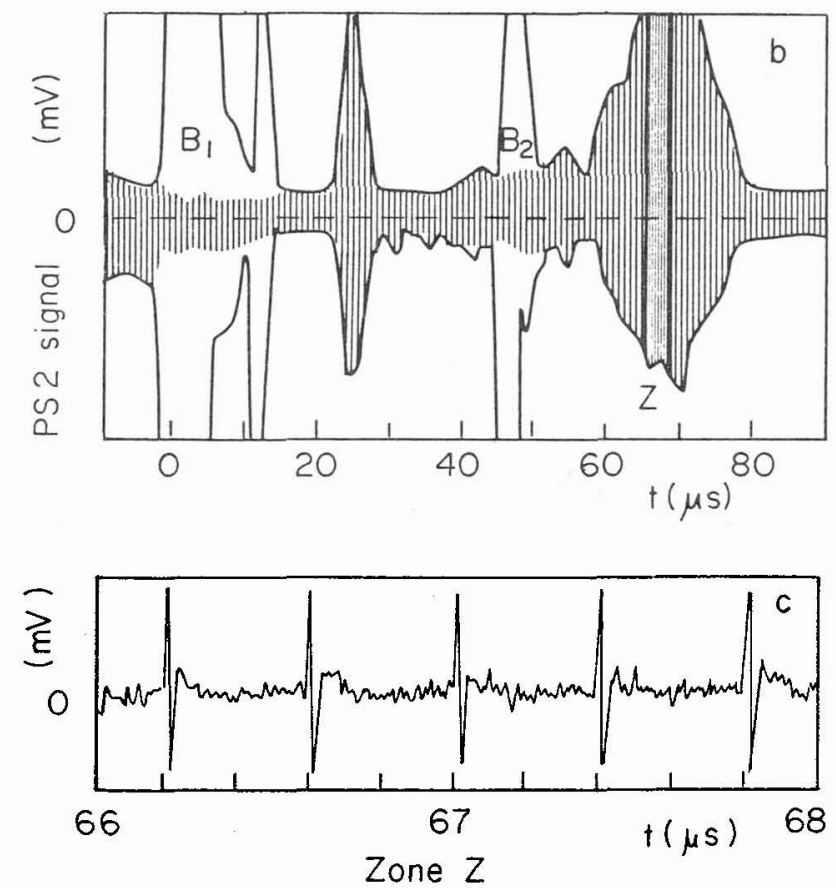

Fig. 4 - Beam transmission through plasma. b - signal from PS2. B1,2 : noise bursts. c - time dilatation of PS2 signal. 
to the charge yield inside a beam pulse. Before ignition of the plasma this amplitude corresponds to the beam transmission in the cold gas. The first ten microseconds correspond to the ignition of the plasma. After a short period during which the noise pick up prevails we observe an attenuation of the phase probe response, indicating a loss of global charge yield in the detected burst by a factor 2. This attenuation has not yet been clearly attributed but reasonable hyoothesis in an enhanced straggling or a defocusing effect which deflected part of the beam out of axis and then reduces the transmission of the beam through the exit collimator.

More interesting are these two time windows during which, on the contrary we can notice an increasing amplitude of the detected signal. This allows us to assume a plasma focusing confiauration during which the transmission through the collimators increase by a factor 4 . A comparison with the evolution of the current flow inside the plasma tube during the discharge, indicates that the transmission windows are correlated with positive alternances of the current. This focusing defocusing effect seems to be linked with the magnetic instabilities generated during the discharge. A more complete understanding of this phenomena is essential to optimize the transmission process. This can also be the source of interesting applications to focus beams if this effect is controlled. This is not yet the case and we need specific experiments to determine magnetic profiles inside the tube and to vary the plasma parameters (geometrical configuration of the electrodes for instance).

Anyway, the only aspect that we have considered in these experiments was the opportunity to realise beam pulse time of flight in good conditions, by selecting the appropriate time period.

\subsection{Stopping power measurements}

Measurements have been made during the two appropriate time window : 20 to $35 \mu s$ and 50 to 75 us. These two regions present plasma characteristics which are completely different. Figure (5) presents the density and temperature evolution during one plasma shot ( $13 \mathrm{KV}$ discharge). After $15 \mu \mathrm{s}$ the electron density reaches a value of $4 \times 10^{17} \mathrm{~cm}^{-3}$ and the temperature amounts to $2.7 \times 10^{4} \mathrm{~K}$. After a rapide decrease the two distributions tend to flatten around values of density and temperature respectively. equal to $2.7 \times 10^{17}$ and $1.8 \times 10^{4} \mathrm{~K}$. (see A. FLEURIER reports). Figure (6) presents the energy loss between vacuum and plasma for an incident sulphur ion, and corresponds to a $13 \mathrm{KV}$ discharge in 9 Torr of Hydrogen. The experimental energy loss (open points) are compared to the calculated values which include :

- Cold gas corrections in the two collimators

- Energy loss calculation which includes several ingredients (Effective $Z$ of the projectile before interaction, density of free electrons and ionisation ratio obtained from SAHA equation or from direct measurements of the neutral atoms density).

A quite good agreement is observed between experimental and calculated values in the region 20 $25 \mathrm{\mu s}$ for which the plasma density and ionisation are rather high. The two dashed 1ines correspond to the limits of uncertainties due mainly to the neutral atom determination ( $50 \%$ uncertainty). In the region 60 - $75 \mu \mathrm{s}$ the difference between $\triangle E$ and $\triangle E C$ is slightiy out of the uncertainty range. This region corresponds to the low density and temperature region where the relative contribution of bound electrons is important. More recent measurements indicate that the cold gas correction has to be estimated very carrefully. We probably underestimate this cold gas correction and a new construction of the interface with the differential pumping system is under study.

Anyway, these measurements present a good evidence which has been confirmed in the Darmstadt experiments of an enhanced stopping power by a factor about two, of the plasma relative to its
equivalent cold matter.

Finaly two comparisons which confort our analysis will be presented : a comparison between a Carbun run and a Sulphur run, and a comparison between two Sulphur runs obta in for two different plasma densities (same time region). Figure (7). The first graph (a) presents time of flight measurements for Carbon and Sulphur experiments. The corresponding stopping power expressed in MeV are $4.3 \mathrm{MeV}$ for the Sulphur ions as it was already mentionned and $0.86 \mathrm{MeV}$ for the Carbon experiment. These two values are roughly in the ratio of the effective charge state (5). Given that the incident velocity is the same for the two incidents ions, this ratio corresponds to the expected behaviour.

Now for the same time window, a comparison between two plasma densities obtained varying the intensity of the electrical discharge : 11 and $13 \mathrm{KV}$ (b). We observe an increasing stopping power which can be compared with the corresponding measured densities and ionisation ratios. The more intense discharge is associated to an energy loss of $4.61 \mathrm{MeV}$, the lower one to $4.26 \mathrm{MeV}$. After cold gas correction and taking into account of the measured ionisation ratio in this time region 


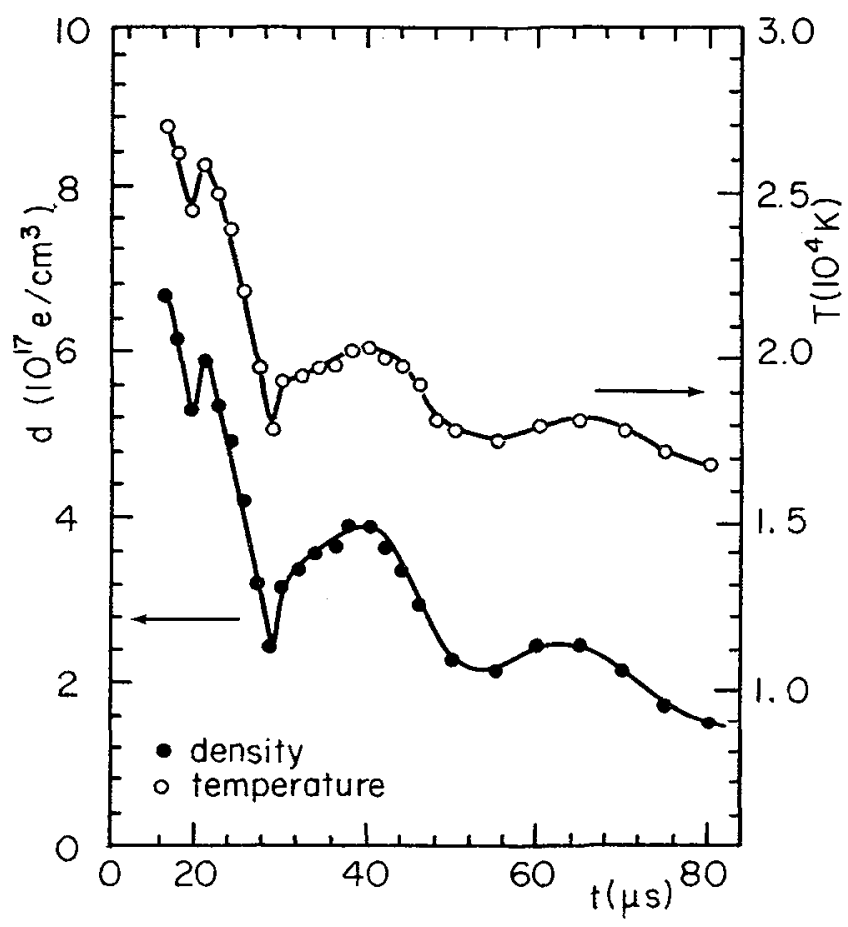

Fig. 5 - Evolution of the electron density (d) and plasma temperature ( $T$ ) versus time ( $t$ ) for a $13 \mathrm{kV}$ discharge in 9 torr $\mathrm{H}_{2}$.

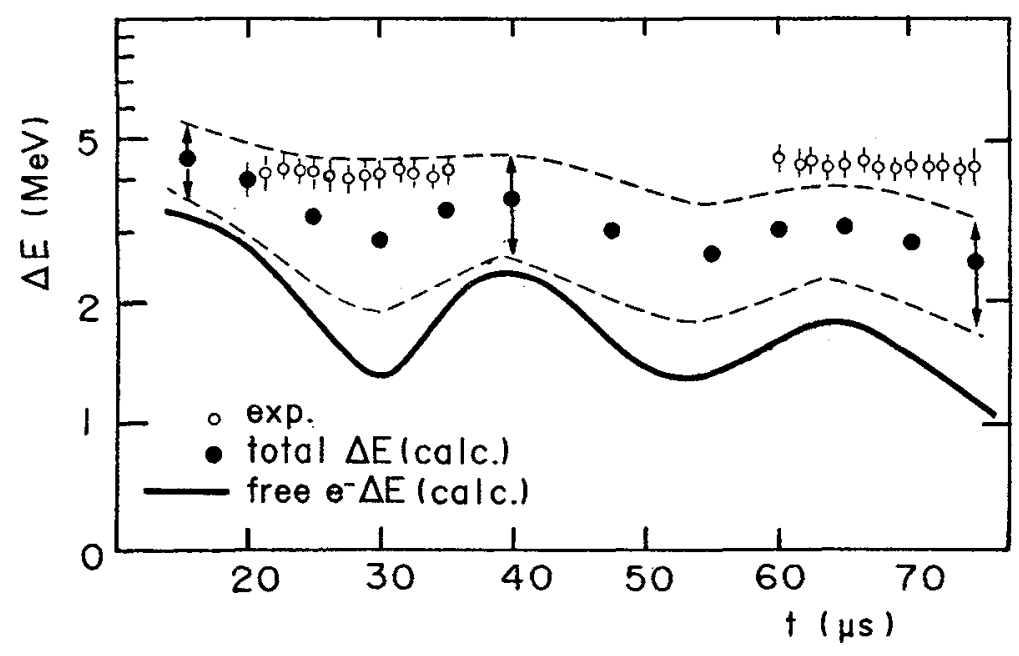

$$
S+\text { Plasma } 13 k V
$$

Fig. 6 - Experimental and calculated energy loss plasma. 
we obtain nearly the same energy loss related to comparable electron density, that is to say: $2.42 \mathrm{MeV}$ and $2.5 \mathrm{MeV}$. This indicates a rather good coherence with the estimation of the plasma parameters.

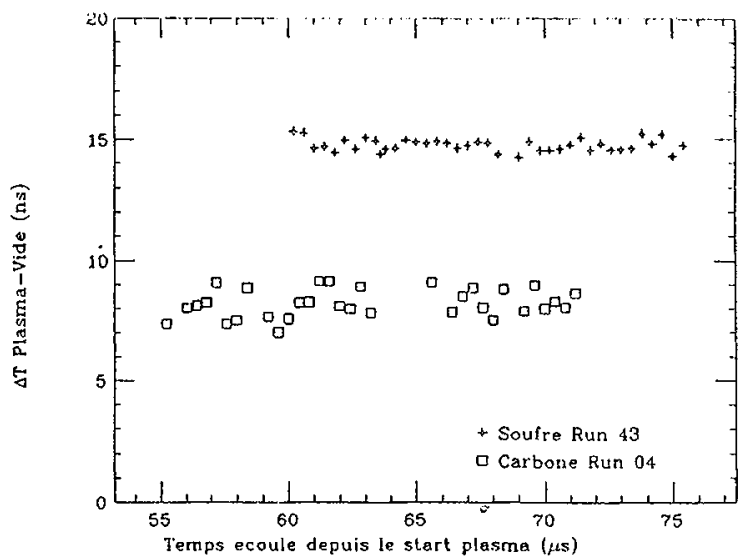

(a)

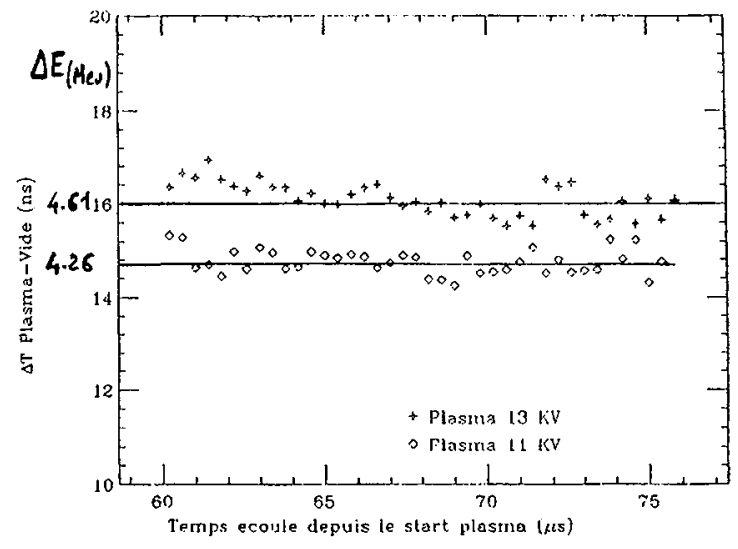

(b)

Fig. 7 - Comparison between $C$ and Sulphur ions for the same incident velocity and plasma characteristics (a) and between 2 plasma configurations (11 and $13 \mathrm{Kv}$ discharge) (6).

\section{4 - CONCLUSION}

In conclusion, our measurements seem, at least for the two density regions, in agreement with estimations based on plasma diagnostics. It remains. some uncertainties mainly due to the cold gas corrections. Our future preoccupations will be to improve the transmission of the beam through the plasma target, by changing geometrical structure of the plasma generator.

However, these experiments open a new way for the investigation of beam-plasma interaction.

\section{REFERENCES}

/1/ R. Bimbot, D. Gardès, S. Della Nagra, M.F. Rivet, A. Servajean, C. Deutsch, E. Maynard, C. Fleurier, A. Sanba, Bulletin Société Française de Physique, Avril $1988 n^{\circ} 68$ p. 18.

/2/ R. Bimbot, S. Della-Negra, D. Gardès, M.F. Rivet, C. Fleurier, B. Dumax, D.H.H. Hoffmann, K. Weyrich, J. Jacoby, H. Wahl, C. Deutsch, G. Maynard, to be published in Laser Int. \& Rel. Plasma Phen., ed. H. Hora, vol. 8, 1988 ; and Rep.. IPN 88-14.

/3/ D.H.4. Hoffmann, K. Weyrich, H. Wah1, Th. Peter, J. Meyer-ter-Vehn, J. Jacoby, R. Bimbot, D. Gardès, M.F. Rivet, M. Dumail, C. Fleurier, A. Sanba, C. Deutsch, G. Maynard, R. Nol1, R. Haas, R. Arnold, S. Maurmann, accepted for publication in Z. Phys. A., 1988.

/4/ D. Gardès, R. Bimbot, S. Della Negra, M. Dumail, B. Kubica, A. Richard, M.F. Rivet, A. Servajean, C. Fleurier, A. Sanba, C. Deutsch, G. Maynard, D.H.H. Hoffmann, K. Weyrich, H. Wahl submitted to Europhys. Letters; IPN0-DRE 88-14.

15/ R.C. Arnold, J. Meyer-ter-Vehn, Rep. Prog. Phys. 50 (1987) 559-606.

i6/ G. Maynard, Thèse Orsay 1987.

17/ C. Fleurier, A. Sanba, J. Mathias, B. Dumax, J.C. Pellicier, G.R.E.M.I. Report (0rléans), nov. 1986.

18/ E. Festa, R. Sellem, Nucl. Iristr. and Meth. 188 (1981) 99. 